\title{
EFEITOS INDESEJÁVEIS DOS HIPOLIPEMIANTES: CONDUTAS NA PRÁTICA CLÍNICA
}

Neusa Forti,Jayme Diament

Trabalho realizado na Faculdade de Medicina da Universidade de São Paulo - Instituto do Coração, São Paulo, SP

*Correspondência

Av. Dr. Enéas de Carvalho

Aguiar, 44

CEP 05403-000

São Paulo - SP

\begin{abstract}
RESUMO
Os fármacos hipolipemiantes, apesar de diminuírem a morbimortalidade por doença coronariana, não são destituídos de efeitos indesejáveis. Estes freqüentemente são transitórios, mas podem ocorrer alterações clínicas e laboratoriais que exigem especial atenção e diferentes condutas. Neste artigo, os autores relatam fundamentalmente como proceder diante do comprometimento muscular e hepático, considerados efeitos adversos mais relevantes dos hipolipemiantes. De modo sucinto, apontam os demais efeitos e a respectiva conduta.
\end{abstract}

Unitermos: Antilipêmicos/efeitos adversos. Miopatia. Hepatotoxicidade.

\section{INTRODUÇÃO}

As dislipidemias, particularmente a hipercolesterolemia, destacamse entre os fatores de risco para doença aterosclerótica. Seu adequado tratamento mostrou-se capaz de diminuir a morbimortalidade dessa entidade nosológica. Entretanto, como todos os medicamentos, os hipolipemiantes não estão isentos de alguns efeitos indesejáveis. Apesar de transitórios, na maioria dos casos, requerem atenção e detecção precoces; às vezes, exigem interrupção do uso. São citados: I) cefaléia, insônia, sintomas gastrintestinais, alterações cutâneas, litíase biliar; 2) alteração da absorção e da metabolização de outros fármacos; 3) aumento das taxas sanguíneas de enzimas musculares (creatinofosfoquinase - CK) e hepáticas (transaminase glutâmico oxalo-acética ou aminotransferase - TGO/AST - e transaminase glutâmico pirúvica ou alaninoaminotransferase - TGP/ALT), da creatinina, da glicose, do ácido úrico; 4) proteinúria; 5) comprometimento muscular, hepático, renal e neurológico!

Após tecer comentários relativos à determinação de taxas enzimáticas, conceito de miopatia e hepatotoxicidade, nesta publicação, abordaremos fundamentalmente a conduta a ser tomada diante dos comprometimentos muscular e hepático induzidos pelos hipolipemiantes em uso clínico. De modo sucinto, relataremos as demais alterações e respectiva conduta.

\section{Alteração de enzima e COMPROMEtimento muscular}

\section{Determinação da creatinofosfoquinase (CK)}

Antes de iniciar o tratamento com hipolipemiantes, solicita-se a determinação da concentração sérica da CK. Se estiver dentro dos valores normais, inicia-se o tratamento de imediato. Mas se os valores excederem três vezes o valor superior da normalidade (VSN), a causa deve ser investigada. Entre as causas, destacam-se o abuso de bebidas alcoólicas, exercícios físicos vigorosos, hipotireoidismo. Em alguns indivíduos, nada é detectado. Afastada a causa, o tratamento pode ser iniciado apesar desses valores.
Durante o tratamento, classicamente se recomenda a determinação de CK após 30, 60, 90, 180, 270 e 360 dias, por ocasião do aumento da posologia e sempre que surgirem sintomas musculares ${ }^{2,3}$.

Recentemente, a força-tarefa da Associação Nacional de Lípides dos Estados Unidos (NLA) questionou se a CK deve ser determinada em todos os pacientes que serão submetidos ao tratamento hipolipemiante e recomendou a determinação somente quando há risco de toxicidade muscular ${ }^{4,5}$.

\section{Alterações musculares e $\mathbf{C K}^{4-9}$}

As alterações musculares são genericamente conhecidas como miopatias. Segundo Pasternak et al. ${ }^{7}$ incluem: I) mialgia ou fraqueza muscular, sem alteração de CK. Também são referidas cãibras, rigidez muscular, sensação de peso, diminuição da força durante a atividade física e até tendinite; 2) miosite, quando os sintomas musculares são acompanhados por aumento de CK até no máximo 10 vezes o VSN; 3) rabdomiólise, quando os sintomas musculares são acentuados, acompanhados por mioglobinúria, sem ou com aumento de CK superior a 10 vezes o VSN e aumento de creatinina sérica. A NLA4, em recente publicação, critica o termo miosite, pois este implicaria em inflamação muscular, o que não acontece com injúria muscular induzida, por exemplo, pela vastatina. Propõe a seguinte classificação: I) miopatia sintomática, que incluiu a dor muscular (mialgia), fraqueza muscular e cãibras; 2) miopatia assintomática, na qual só há aumento de CK; 3) rabdomiólise clinicamente importante com: a) aumento discreto de CK (CK até 10 vezes o VSN); b) aumento moderado de CK (superior a 10 vezes, mas inferior a 50 vezes o VSN); c) aumento acentuado de CK (superior a 50 vezes o VSN).

A sintomatologia muscular geralmente surge no início do tratamento, mas pode aparecer no seu decorrer, mesmo após anos. Relacionase às taxas séricas dos hipolipemiantes, usados isoladamente ou associados, e também ao emprego concomitante de outros fármacos.

Várias hipóteses têm sido aventadas para explicar o comprometimento muscular decorrente dos hipolipemiantes: I) instabilidade da 
FORTI N ET AL.

membrana do musculoesquelético conseqüente à diminuição de colesterol; 2) diminuição da produção do farnesil-pirofosfato (principalmente induzido pelas vastatinas), substância intermediária para a síntese de ubiquinona (coenzima QI0), necessária para o transporte de elétrons e fosforilização oxidativa da mitocôndria; 3) redução das "GTP-binding-proteínas" (Rho, Ras, Rac), conseqüente à ação das vastatinas e que exercem importante papel na proliferação e crescimento celular; 4) interrupção da isopentenilação da selenocisteína tRNA pelas vastatinas; 5) inibição da glucuronidaçã̃o das vastatinas pelos fibratos, em particular pela genfibrozila ${ }^{10,11}$. Recentemente, postulouse que a miopatia provocada por vastatina estaria vinculada à determinação genética: variações nos genes $\mathrm{COQ2}$ levariam a alterações de vias envolvendo a ubiquinona ${ }^{12}$.

\section{Vastatinas $^{\mid 1,4-7,10,11,13,14}$}

Em investigações clínicas relacionadas à eficácia e segurança das vastatinas e em estudos em prevenção primária e secundária de coronariopatia aterosclerótica, a freqüência de alterações musculares foi inferior a $1 \%$. Recentes publicações evidenciaram: I) por análise de 14 ensaios randomizados, envolvendo 90.056 participantes, que 0 risco de miopatia é dose dependente e que a ocorrência de rabdomiólise é extremamente rara (excesso de risco 0,01\% em cinco anos) ${ }^{13} ; 2$ ) em 18 estudos que incluíram 36.062 pessoas tratadas com vastatinas e 35046 com placebo, o grupo tratado apresentou significante maior número de casos com aumento de CK (8I vs 64) e de alterações musculares (316 vs 253); a rabdomiólise ocorreu em nove casos: três com sinvastatina, um com lovastatina e cinco com placebo ${ }^{14}$; 3) em estudos randomizados, para cada 100 mil pessoas/ ano, a incidência de miopatia foi igual a I I e o de rabdomiólise foi igual a $3,4^{\prime \prime} ; 4$ ) considerando as vastatinas em uso clínico (com exceção da rosuvastatina), a freqüência de miopatia foi menor com as vastatinas hidrofilicas (fluvastatina e pravastatina) e maior nos tratados com as lipofilicas (atorvastatina e sinvastatina) ${ }^{14}$; a rabdomiólise é extremamente rara $(1: 1000.000)$, mas a cerivastatina provocou acentuado número de casos, (cinco a sete vezes mais) e foi retirada do comércio em 200 |5,11.

Em um grupo de idosos (média 69 anos), tratados com sinvastatina durante 60 meses, observaram-se elevações discretas de CK somente em três casos ${ }^{15}$. Em hipercolesterolêmicos (média $55 \pm 7,8$ anos) tratados com sinvastatina, após 30 e 60 dias, não foram verificadas modificações de parâmetros isocinéticos e de conduções nervosa e sensitiva ${ }^{16}$.

É oportuno lembrar que a lovastatina, a sinvastatina e a atorvastatina são metabolizadas no sistema enzimático CYP P 4503 A4, a fluvastatina no CYP P 450 2C9 e 2D6 e a rosuvastatina no CYP P 450 $2 \mathrm{C} 9$ e $2 \mathrm{Cl}$; ; a pravastatina é metabolizada preferencialmente por sulfataçãa. A concentração sérica das vastatinas pode elevar-se pela administração concomitante de medicamentos ou substâncias que utilizam o CYP P 450 3A4 para metabolização ou que o inibam (eritromicina, anti-retrovirais, anti-fúngicos, ciclosporina, verapamil, suco de grape-fruit, por exemplo). O mesmo pode ocorrer em relação ao CYP P 450 2C (amiodarona, cimetidina, fluoxetina, fenitoína, varfarin, por exemplo)!.
Especialistas da NLA4 concluíram que: I) não há evidências para admitir com convicção que as diferentes alterações musculares sejam manifestações do mesmo processo patológico, elas seriam causadas por diferenças individuais na suscetibilidade, tolerância a dor e outros fatores; 2) a miopatia e a rabdomiólise estão associadas a: a) efeito de classe; b) doses mais elevadas; c) a maiores níveis séricos; d) provavelmente à lipoflicidade, pois a hidroflicidade diminui a entrada da vastatina no músculo, diminuindo o dano muscular; e) associação de fármacos que utilizem o CYP P 3503 A4 como substrato ou que o inibem; f ao aumento da meia-vida, por alteração da glucuronidação; 3) a miopatia e a rabdomiólise não dependem do grau de redução de LDL-C; 4) o risco de miopatia e rabdomiólise é maior em a) idosos, principalmente acima dos 70 anos; b) indivíduos com hipotiroidismo, função renal alterada (clearance de creatinina $<30 \mathrm{mg} / \mathrm{dl}$ ), função hepática alterada, proteinúria pp. em diabéticos); c) quando há uso de drogas que utilizam o CYP P 450 3A4 como substrato ou que o inibem; d) durante doença aguda ou cirurgia; e) em indivíduos que praticam exercícios vigorosos (corredores de maratona por exemplo); f níveis basais de CK entre dois a cinco vezes - VSN. Em relação à associação com outros hipolipemiantes, o risco aumenta quando se associam fibratos (principalmente genfibrozila) ou ácido nicotínico de liberação lenta (SR). Não aumenta se associados resinas, ezetimiba, ômega 3, fitosteróis e fitostanóis. Chamam a atenção para o fato de que os níveis elevados de CK isoladamente são indicativos de dano muscular e que fraqueza e dor muscular, independente dos níveis de CK, também indicam danos musculares. Não recomendam biópsia para esclarecer o diagnóstico.

$\mathrm{Na}$ prática clínica, devemos ter em mente que as vastatinas podem induzir aumento dos níveis séricos de CK, independentemente de ocorrer sintomatologia muscular. Esta também ocorre com ou sem alteração de CK. A conduta seguinte é recomendada:

a) CK e ausência de sintomas musculares

Se os valores de CK estiverem aumentados até três vezes o VSN, após investigar causa desencadeante e, se possível corrigíla, a determinação de CK deve ser repetida após quatro a seis semanas. Se mantidos até três vezes o VSN, prossegue-se o tratamento. Caso os valores se encontrem entre três a dez vezes o VSN e/ou se elevarem progressivamente, porém discreta ou moderadamente, procurar identificar a causa desencadeante e corrigir se possível. A determinação de CK é repetida após quatro a seis semanas. Caso persistam elevados (três a dez vezes 0 VSN) reduz-se a dose da vastatina ou substituímos por vastatina que não utilize o CYP P 4503 A4 ou por outra classe de hipolipemiante (resinas seqüestrantes de ácidos biliares, ezetimiba por exemplo). Quando os valores de CK forem superiores a dez vezes o VSN e/ou a elevação é progressiva e acentuada, interrompe-se a terapêutica com vastatina e repetem-se as determinações de CK mais amiúde. Paralelamente, procurar identificar sintomatologia muscular. Caso ocorra normalização, recomenda-se a introdução de vastatina não metabolizada no CYP P 450 3A4 ou substituição por outra classe de hipolipemiante;

b) CK e sintomas musculares presentes

Se os valores de CK estiverem elevados até três vezes o VSN ou mesmo próximos da normalidade, após investigar se há outra causa desencadeante, repete-se a determinação dentro de quatro a seis 
semanas. Caso os níveis sejam mantidos e a sintomatologia muscular não for importante, diminui-se a dose de vastatina ou procede-se sua troca por outra de menor potência de ação e não metabolizada no CYP P 450 3A4; o emprego de outra classe de hipolipemiante também deve ser aventado. Quando os valores de CK se encontrem entre três a dez vezes o VSN, interrompe-se a terapêutica com vastatina, investiga-se outra causa desencadeante, repete-se a determinação no máximo após quatro semanas. Se os valores se mantiverem entre três a dez vezes ou mesmo inferiores a três vezes o VSN, reduz-se a dose, ou substituímos por outra que não utilize o CYP P 4503 A4 ou por outra classe de hipolipemiante. Caso os valores de CK excederem dez vezes o VSN, interrompe-se de imediato a administração da vastatina, avalia-se a presença de mioglobinúria e elevação das taxas séricas de creatinina, alcaniza-se a urina e hidrata-se o paciente. Quando normalizarem, reinicia-se a terapêutica com outra classe de hipolipemiante.

Considerando a sintomatologia muscular, os especialistas da NLA $A^{4,5}$ recomendam: I) se os sintomas musculares forem intoleráveis, com ou sem elevação das taxas de CK, interrompe-se o uso da vastatina. Quando desaparecerem, reintroduzir na mesma dose ou em doses mais baixas. Se houver recorrência, descontinuar a vastatina e administrar outra classe de hipolipemiante. 2) se os sintomas forem toleráveis ou se os pacientes são assintomáticos com CK elevada até 10 vezes o VSN, prosseguir o tratamento na mesma dose ou em dose menor. A sintomatologia é utilizada como orientação para interromper ou continuar com a terapia; 3) interrupção imediata da administração de vastatina se ocorrer rabdomiólise, e, se necessário, hospitalizar o paciente. Após recuperação, avaliar o emprego de outra classe de hipolipemiante.

Até o momento, não há evidências para recomendar o uso rotineiro da coenzima Q10 no tratamento das miopatias induzidas pelas vastatinas, apesar de relatos de diminuição da dor ${ }^{17,18}$.

\section{Fibratos $^{1,9,19}$}

Os fibratos também induzem o aparecimento de miopatia, embora raramente. A miopatia é mais freqüente com a genfibrozila, principalmente se associada à vastatina, provavelmente por inibir a glucuronidação desta e acentuar a desestabilização da membrana celular. O risco é mais acentuado em diabéticos, renais crônicos, hipotireóideos e idosos.

Os cuidados para evitar os sintomas e tratar a dislipidemia são semelhantes aos já descritos para as vastatinas. Lembramos que os fibratos utilizam preferencialmente para metabolização as izoenzimas 2C9 e 2CI 9 do CYP P 450.

\section{Ácido nicotínico $1,9,20$}

O ácido nicotínico, quando associado a vastatina, facilita o desencadear de miopatia, exigindo controles periódicos de CK e cuidados já apontados.

\section{Alteração de enzimas e comprometimento hepático}

\section{Determinação de enzimas hepáticas}

Antes de iniciar o tratamento, determinam-se os valores da TGO/ AST e TGP/ALT. Caso seus valores excedam três vezes o VSN, o tratamento pode ser iniciado, após excluir doença hepática em atividade e abuso de bebidas alcoólicas. Durante a terapêutica hipolipemiante, classicamente é recomendada a determinação das taxas das enzimas hepáticas após 30, 90, 180, 270 e 360 dias e por ocasião do aumento da posologia e quando houver sensação de malestar, fadiga ou icterícia 2,3 . A NLA recomenda determinação somente antes e após 12 semanas ou quando houver aumento da dose ${ }^{5}$.

\section{Comprometimento hepático $0^{5,7-9,21}$}

Os hipolipemiantes podem ocasionar toxicidade hepática que se manifesta, na clínica, de diferentes formas: elevação assintomática das enzimas TGO/AST, TGP/ALT, insuficiência hepática aguda, hepatite e colestase. Também é relatado o aumento da litogenicidade da bile relacionado principalmente ao emprego de fibratos. Lembramos que alterações de enzimas hepáticas ocorrem também na hepatite crônica, figado gorduroso ou esteatose hepática de etiologia não-alcoólica e não contra-indicam o emprego dos hipolipemiantes e nem a interrupção de seu uso, mas exigem maior atenção do clínico. Conduta semelhante deve ser observada em relação ao consumo moderado de álcool (Um a dois "drinks"/dia).

Diferentes mecanismos são aventados para explicar o comprometimento hepático: I) alteração da homeostase do cálcio intracelular decorrentes de reações que envolvem o CYP P 450, elevando a lise celular; 2) alteração nas proteínas de transporte relacionada com o fluxo de ácidos biliares (por exemplo, com fibratos); 3) formação de metabólitos no fígado decorrentes de reações imunológicas; 4) indução de resposta citolíticas por células $T$; 5) apoptose por agressão imunomediada; 6) estresse oxidativo gerado por danos e organelas intracelulares ${ }^{21}$. Chang e Schiano ${ }^{22}$ sugerem que polimorfismos genéticos envolvendo o metabolismo das drogas explicariam a diferença de suscetibilidade individual à hepatotoxicidade.

Predispõem à hepatotoxicidade: idade avançada, hipoglicemiantes orais, excesso de bebidas alcoólicas, doses elevadas dos hipolipemiantes.

\section{Vastatinas $^{5,7-9,11,13,21}$}

São poucos conhecidos os mecanismos pelos quais as vastatinas provocam hepatotoxicidade; talvez mecanismos conjugados estejam envolvidos. Aproximadamente $1 \%$ dos indivíduos tratados com vastatina apresentam elevação das taxas séricas das enzimas hepáticas nos primeiros três meses de tratamento, muitas vezes transitória e/ou dose dependente, mas relacionada a efeito de classe. Durante o tratamento, doença hepática preexistente, colestase e o abuso de bebidas alcoólicas favorecem o aumento dessas enzimas. Porém, não existem evidências de que esse aumento anteceda o desencadear de insuficiência hepática aguda: esta é extremamente rara e sua notificação é de I: I .000.000 pessoas/ano tratadas. Colestase e hepatite ocorrem muito raramente e não foram relatadas nos grandes ensaios clínicos. Também é rara a hepatite auto-imune ${ }^{23}$.

Se durante o tratamento com vastatina ocorrer elevação das enzimas hepáticas até 3 vezes o VSN, repete-se sua determinação após seis a 12 semanas. $O$ abuso de bebidas alcoólicas e possível interação medicamentosa devem ser pesquisados. Se os valores se normalizarem e se mantiverem, prossegue-se o tratamento, tomando-se maiores precauções com o aumento da dose. Quando os valores das enzimas 
FORTI N ET AL.

hepáticas excederem 3 vezes o VSN, repete-se a determinação das taxas em uma semana, verifica-se o abuso de álcool e interação medicamentosa, procura-se identificar a doença hepática em atividade. Persistindo o aumento, repete-se novamente após duas a quatro semanas, diminui-se a dose da vastatina ou mesmo, interrompe-se o seu uso. Se normalizar ou diminuir, reintroduz-se vastatina, de preferência não metabolizada no CYP P 450 3A4 ou emprega-se outra classe de hipolipemiante. Caso o aumento das taxas enzimáticas seja acompanhado por icterícia, o tratamento com vastatina deve ser imediatamente suspenso.

Não há evidências de prejuízo na terapia anticoagulante relacionada à elevação de enzimas hepáticas.

\section{Fibratos $1,9,19$}

Os fibratos são litogênicos por aumentar a secreção de colesterol para a bile, sem alterar as secreção de ácidos biliares ou fosfolípides resultando em aumento da saturação do colesterol na bile. Estudos epidemiológicos evidenciaram que o risco relativo para coledocolitíase em pacientes tratados com fibratos é de 1,7 vezes e é mais freqüente com clofibrato.

Recomenda-se cuidadosa anamnese para identificar possíveis indivíduos portadores de litíase biliar antes de iniciar o tratamento. Se durante a terapêutica houver alguma manifestação de litíase, confirmar por ultrassonografia, suspender o fibrato, substituir por outra classe de hipolipemiante. Não há aumento de litogenicidade quando se associa vastatina, pois esta diminui a secreção biliar de colesterol.

Também são descritas discretas elevações de enzimas hepáticas, e a conduta é semelhante à relatada para as vastatinas.

\section{Ácido nicotínico 19,20}

O ácido nicotínico provoca alteração hepática grave, principalmente na forma SR (liberação lenta, sustentada), tornando imperioso o controle periódico das taxas séricas das enzimas hepáticas: determinação a cada seis a 12 semanas nos primeiros seis a 12 meses de terapia e depois a cada seis meses.

Indica-se a diminuição da dose de ácido nicotínico ou a interrupção do tratamento, se ocorrer elevação dos níveis séricos das enzimas hepáticas até três vezes o VSN, especialmente se associada à elevação das bilirrubinas, do tempo de protrombina e de sintomatologia. A terapêutica deve ser imediatamente interrompida caso a elevação das enzimas ultrapassar três vezes o VSN, prosseguindo o tratamento com outra classe de hipolipemiante. Aumento isolado de bilirrubina (<3mg/ dl) não requer diminuição da dose ou interrupção do tratamento, pois é indicativo de interferência no transporte do da bilirrubina.

\section{Efeitos INDESEjÁVEIS SOBRe 0 SISTEMA Digestivo de HIPOLIPEMIANTES COM AÇÃO INTESTINAL $1,9,24$}

\section{Resinas seqüestrantes de ácidos biliares}

Estes fármacos têm sabor desagradável e para ingeri-los, recomenda-se a diluição em sucos. Diminuem a absorção de vitaminas (A,D,K,E) e de ácido fólico, exigindo, as vezes, suplementação principalmente em crianças. Diminui também a absorção de outros fármacos, como, digitálicos e anticoagulantes, fenilbutozona, diuréticos tiazídicos, penicilina, tetraciclina, hormonio tireoideanos, estrógenos, progestágenos. Se necessário esses medicamentos devem ser administrados I hora antes ou três horas após a resina. Com o colesevelam os efeitos são bem menos acentuados. Elevam a trigliceridemia, exigindo, às vezes, a associação em fibratos. Não alteram as enzimas hepáticas.

\section{Ezetimiba}

Recentemente introduzida no comércio, pode provocar distúrbios gastrintestinais, raramente mialgia (por provável alteração da membrana celular) e discretas elevações de enzimas hepáticas.

\section{Alterações renais}

\section{Vastatinas $^{5,11,25}$}

Aumento dos valores séricos de creatinina tem sido relatado com o uso de vastatina, mesmo na ausência de rabdomiólise. A insuficiência renal ocorre em cada 0,3 a 0,9 casos por 1.000.000 de prescrições. Se confirmado o incremento da creatinina sérica, a dose é ajustada e o paciente encaminhado ao nefrologista. Doença renal crônica não contra-indica o seu emprego, embora as doses devam ser ajustadas e dado preferência a fluvastatina.

Também tem sido descrita proteinúria, particularmente, com rosuvastatina na dose de $80 \mathrm{mg} /$ dia. Estudos experimentais apontam para bloqueio da absorção tubular de proteína pela vastatina. Após investigar a causa da proteinúria e ajustar a dose, solicitar a opinião do nefrologista.

\section{Fibratos 1,19}

Os fibratos ativam os PPARS, prejudicam a geração de prostaglandinas vasodilatadoras, aumentam a produção de creatinina sem provocar dano renal. Esse aumento é moderado e reversível.

A creatinina sérica deve ser determinada antes de administrar um fibrato. Se os valores estiverem discretamente aumentados, prefere-se a genfibrozila ou fenofibrato em doses baixas. Caso a creatinina se eleve durante o emprego do fibrato, repetem-se dosagens, diminui-se a dose, dando-se preferência pela gentibrozila ou mesmo substituindo por outra classe de hipolipemiante.

Atenção maior deve ser dada a administração concomitante de outros fármacos capazes de alterar a função renal como a metformina. Maiores cuidados devem ser tomados quando a função renal já está alterada, pois os fibratos são eliminados preferencialmente por via renal; neste caso, empregar doses menores e determinar a creatinina mais freqüentemente.

\section{Alterações do sistema nervoso 11,26}

Recentemente, descreveram-se raros casos de neuropatia periférica em indivíduos tratados com vastatinas. Nestes casos é imperioso investigar outras causas de neuropatia periférica como diabete melito, insuficiência renal, abuso de bebidas alcoólicas, deficiência de vitaminas BI2, neoplasia, síndrome de imunodeficiência, intoxicação por metal pesado. Afastadas essas causas, interrompe-se o uso da vastatina por três a seis meses. Desaparecendo os sintomas reintroduz-se o tratamento de preferência com classe de hipolipemiante. Estudos como o HPS $^{27}$ e PROSPER ${ }^{28}$ não mostraram relação entre vastatinas e neuropatia periférica. 
Não se evidenciou relação entre o uso de vastatina e prejuízo da função cognitiva. Aliás, no HPS, houve melhora de função cognitiva ${ }^{27}$. Resultado semelhante foi encontrado em pequeno estudo em pacientes com Alzheimer ${ }^{29}$.

\section{Alterações CutÂneas $\mathbf{S}^{1,20}$}

O "flushing" e o "rash" cutâneo constituem os efeitos indesejáveis de particular importância relacionados ao emprego do ácido nicotínico.

O "flushing" é causado pela liberação de prostaglandinas e outros eicosanóides das células para a pele. Não é de natureza alérgica, ocorre em quase todos os indivíduos tratados com niacina de ação rápida (IR) e constitui a maior causa de descontinuidade de tratamento. É exacerbado por bebidas alcoólicas, alimentos apimentados, bebidas e banhos quentes. Corrigidos esses fatores, para diminuir o "flushing", preconizam-se a administração de aspirina 30 minutos antes da niacina e o emprego de niacina de ação intermediária (FR).

O "rash" cutâneo usualmente acompanha o "flushing", é transitório, mas pode, às vezes, persistir entre uma dose e outra; é acompanhado por prurido e, muitas vezes, confundido com urticária. Se houver "rash", diminuir a dose ou suspender a administração.

\section{Outros efeitos INDESEjÁvels $1,19,20$}

Os fibratos e o ácido nicotínico aumentam o tempo de protrombina e o INR. Assim, esses índices devem ser determinados durante 0 tratamento com o hipolipemiante se o paciente usar anticoagulante. Ajustes das doses são requeridos. Conduta semelhante é adotada se o paciente for diabético ou usar hipoglicemiantes.

O ácido nicotínico, além de induzir aumento da glicemia e da hemoglobina glicada ( por aumentar a resistência à insulina), provoca aumento da uricemia, visão borrada, náusea, exarcerbação de úlcera péptica. Dependendo da sintomatologia e do grau de alterações, interromper a administração.

\section{ConClusÃo}

Nos últimos anos, grandes ensaios clínicos e terapêuticos evidenciaram o benefício do tratamento das hiperlipidemias na prevenção da doença aterosclerótica, em particular das coronárias.

Entretanto, apesar de bem tolerados, os fármacos hipolipemiantes apresentam efeitos indesejáveis, quer quando usados isoladamente, quer quando associados relacionados ou não à interaçãa medicamentosa. Às vezes, esses efeitos alcançam grande repercussão clínica.

Conhecer esses efeitos, solicitar exames laboratoriais para melhor identificá-los e saber controlá-los constituem premissas básicas para avaliar o risco benéfico para cada paciente e atingir a meta preconizada pelas atuais Diretrizes ${ }^{30-32}$.

\section{Conflito de interesse: não há}

\section{SUMMARY}

\section{AdVERSE EFFeCtS OF hypolipemic DRUGS: HOW tO TREAT IN CLINICAL PRACTICE}

Hypolipemic drugs improve coronary morbidity and mortality and appear to be safe; nevertheless appropriate monitoring is recommended. Adverse effects are reported that are frequently transitory. Severe adverse effects are infrequent, but clinicians must correctly screen them; symptoms and laboratory changes must be carefully interpreted. Often they call for special treatment and replacement of the hypolipemic drugs in use. This article emphasizes how to treat dyslipidemia if skeletal muscle and liver involvement are present. Briefly other adverse effects are also reported. [Rev Assoc Med Bras 2008; 54(4): 357-62]

KEY WORDS: Hypolipemic, safety. Myopathy. Hepatotoxicity.

\section{REFERÊNCIAS}

I. Forti N, Diament J. Fármacos hipolipemiantes. Rev Soc Cardiol Estado de São Paulo. 2005, 1 5:495-504.

2. Faludi AA, Bertolami MC. Estratégia no seguimento a longo prazo de pacientes dislipidêmicos sob tratamento farmacológico Rev Soc Cardiol Estado de São Paulo. 2005; 1 5:546-9.

3. Martinez TLR, Nascimento HM, Fernandes SC. Investigação inicial e seguimento ambulatorial. In: Fonseca FAH. Doenças cardiovasculares: terapêutica clinica. São Paulo: PlanMark; 2006. v. I, p. I03-6.

4. Thompson PD, Clarckson PM, Rosenson RS. An assessment of statin by muscle experts. Am J Cardiol. 2006;97(Suppl):69C-76C.

5. Mc Kenney JM, Davidson MH, Jacobson TA, Guyton JR. Final recommendations of the National Lipid Association Statin Safety Assessment Task Force. Am J Cardiol. 2006;97(Suppl):89C-94C.

6. Diament J, Forti N, Giannini SD. Hipolipemiantes ealterações musculares. Rev.. Soc Cardiol Estado de São Paulo. 1998;8: I074-80.

7. Pasternak RC, Smith SC, Bairey-Merz CN, Grundy SM, Cleeman JI, Lenfant $\mathrm{C}$. ACC/AHA/NHLBI clinical advisory on the use and safety of statins. Circulation. 2002; I 06: 1024-8.

8. Vacanti LJ, Sposito AC. Contra indicações e efeitos adversos dos hipolipemiantes. In: Fonseca FAH. Doenças cardiovasculares: terapêutica clínica. São Paulo: Planmark; 2006. v. I, p. I06-12.

9. Forti N, Diament J. Mialgia ou elevação de enzimas musculares e hepáticas durante terapêutica hipolipemiante. In:Fonseca FAH. Doenças cardiovasculares: terapêutica clinica. São Paulo, Planmark, 2007. v.2, p 615-9.

10. Vaughan CJ, Gotto AM. Update on statins 2003. Circulation. 2004; I 10:886-92

II. Law M, Rudnick AR. Statin safety: a systematic review. Am J Cardiol. 2006;97:(Suppl I):S52 -S60.

12. Oh J, Ban MR, Miskie BA, Pollex RL, Hegele RA. Genetic determinants of statin intolerance. Lipids Health Dis. 2007;21:6-7.

13. Cholesterol Treatment Trialist'S (CTT) Colaborators. Efficacy and safety of cholesterol-lowering treatment: prospective meta-analysis of data from 90056 participants in 14 randomised trials of statins. Lancet. 2005;366: 1267-78

14. Silva MA, Swanson AC, Gandhi P, Tataronis GR. Statin-related adverse events: a meta analysis. Clin Ther. 2006;28:26-35.

15. Giannini SD, Gois JM, Dereviacki BE. Simvastatin in the treatment of elderly patients with primary hypercholesterolemia. Curr Ther Res. 1994;55:161-7|.

16. Giannini SD, Scholz J, Shinzato GT, Battistela LR, Forti N, et al. Muscular response to mechanical overload in hypercholesterolemic patients treated with simvastatin: isokinetic evaluation through computerized dynamometer: Curr Ther Res. 1996;57:376-89.

17. Marcoff F, Thompson PD. The role of coenzime Q 10 in statin-associated myopathy. J Am Coll Cardiol. 2007:49:2231-7.

18. Caso G, Kelly P, Mc Nurlan MA, Lawson WE. Effect of coenzime Q I 0 on myopathic symptoms in patients treated with statins. Am J Cardiol. 2007:99: 1 409-12.

19. Davidson MH, Armani A, Mc Kenney JM, Jacobson TA. Safety considerations with fibrate therapy. Am I Cardiol. 2007;99(Suppl):S3-SI 8 . 
FORTI N ET AL.

20. Guyton JR, Bays HE. Safety considerations with niacin therapy. Am J Cardiol. 2007;99(Suppl I):S22-S3 I

21. Cohen DE, Anania FA, Chalasani N. An assessment of statin by hepatologists. Am J Cardiol. 2006;97(Suppl):77C-8I C.

22. Chang CY, Schiano TD. Review article: drug hepatotoxicity. Aliment Pharmacol Ther. 2007;25: I |35-5 I.

23. Alla $\vee$, Abraham j, Siddiqui J. et al. Autoimmune hepatitis triggered by statins. J Clin Gastroenterol. 2006;40:757-6I .

24. Jacobson TA, Mc Kenney JM, Guyton JR Safety consideration with gastrientestinally active lipid-lowering drugs. Am J Cardiol. 2007;99(Suppl):47C-55C.

25. Kasiske BL, Wanner C, O'Neill WC. An assessment of statin safety by nephrologists. Am J Cardiol. 2006;97(Suppl):82C-5C.

26. Brass LM, Alberts MJ, Sparks L. An assessment of statin safety by neurologists Am J Cardiol. 2006;97(Suppl):86C-8C.

27. Heart Protection Study Collaborative Group. Effects of cholesterol lowering with simvastatin on stroke and other major vascular events in 20536 people with cerebrovascular disease or other high-risk conditions. Lancet. 2004;363:757-67.

28. Shepherd J, Blauw GJ, Nurphy MB, Bollen EL, Buckley BM, Cobbe SM, et al for the PROSPER study group. Pravastatin in Elderly Individuals at Risk of Vascular Disease (PROSPER): a randomised controlled trial. Lancet. 2002;360: 1623-30.

29. Sparks DL, Sabbagh MN, Connor DJ, Lopes J, Launer LJ, Browne P, et al. Atorvastatin for the treatment of mild to moderate Alzheimer disease. Arch Neurol. 2005;62:753-7.

30. Executive Summary of the Third Report of National Education Program (NCEP). Expert Panel on detection, evaluation end treatment of high blood cholesterol in adults (Adult Treatment Panel III) JAMA. 2001;285:2486-97.

31. Grundy SM, Cleeman JI, Merz CN, Brewer HB Jr, Clarck LT, Hunninghak $\mathrm{DB}$, et al for the NHLBI/ ACC/AHA. Implications of recent trials for the National Cholesterol Education Program Adult Treatment Panel III Guidelines. Circulation. 2004; I 10:227-39.

32. IV Diretriz Brasileira sobre Dislipidemias e prevenção da aterosclerose. Arq Bras Cardiol. 2007;88 (Supl I):2-19.

Artigo recebido: 22/10/07

Aceito para publicação: 01/02/08 